pylori and immune thrombocytopenic purpura: unsolved questions and controversies. Int. J. Hematol. 84:309-315.

22. Veri, M.C., et al. 2007. Monoclonal antibodies capable of discriminating the human inhibitory Fcgamma-receptor IIB (CD32B) from the activating Fcgamma-receptor IIA (CD32A): biochemical, biological and functional characterization. Immunology. 121:392-404.

23. Ghevaert, C., et al. 2007. Management and outcome of 200 cases of fetomaternal alloimmune thrombo- cytopenia. Transfusion. 47:901-910

24. Roberts, I., and Murray, N.A. 2008. Neonatal thrombocytopenia. Semin. Fetal Neonatal Med. 13:256-264.

25. Armour, K.L., Clark, M.R., Hadley, A.G., and Williamson, L.M. 1999. Recombinant human IgG molecules lacking Fcgamma receptor I binding and monocyte triggering activities. Eur. J. Immunol. 29:2613-2624.

26. Armstrong-Fisher, S., et al. 2004. In vitro maternofetal tranfer of native and $\mathrm{Fc}$-mutated recombinant
RhD antibodies [abstract]. Vox Sang. 87:37.

27. Bussel, J., et al. 2006. GMA161 treatment of refractory ITP: efficacy of Fcgamma-RIII blockade [abstract]. Blood (ASH Annual Meeting Abstracts). 108:1074.

28. Bussel, J.B., Schindler, A.M., and Grossbard, E.B. 2007. R935788: A phase II, single center, open label efficacy and safety ascending dose pilot study for the treatment of adult immune thrombocytopenic purpura [abstract]. Blood (ASH Annual Meeting Abstracts). 110:1310.

\title{
Fifty years later: the disk goes to the prom
}

\author{
Mark E. Kleinman ${ }^{1}$ and Jayakrishna Ambati ${ }^{1,2}$
}

1Department of Ophthalmology and Visual Sciences and 2Department of Physiology, University of Kentucky, Lexington, Kentucky, USA.

\begin{abstract}
Although age-related macular degeneration is the most prevalent macular disease in the world, numerous discoveries regarding the molecular bases of vision have been made through genetic association studies of rare inherited maculopathies. In this issue of the JCI, Yang et al. present a functional genetics study that identifies a role for prominin 1 (PROM1), best known as a stem cell and/or progenitor cell marker, in the biogenesis of retinal photoreceptor disk arrays (see the related article beginning on page 2908). This study supports an established model in which disk morphogenesis occurs through membrane evagination and extends other recent studies assigning PROM1 important functions outside of the stem cell niche.
\end{abstract}

\section{Essentials of photoreceptor organization}

More than 50 years ago, the first ultrastructural evidence of photoreceptor disk organization was published by noted electron microscopist Fritiof Sjöstrand (1). Subsequent studies provided more detailed characterizations of the evolutionarily conserved arrangement of rod and cone photoreceptors into inner and outer segments within Bilateria (2). It is in this outer segment region that thousands of rhodopsin-containing bilayered disks form an array of photovoltaic cells that transmit visual stimuli to the neural retinal components. Without the organized development and maintenance of these precious subcellular elements, the eye cannot fulfill its raison d'être.

Many congenital and acquired diseases that result in vision loss are caused by photoreceptor degeneration. The most widely studied of these pathologies is age-related macular degeneration (3), an epidemic in the developed world affecting approxi-

Nonstandard abbreviations used: PROM1, prominin 1 .

Conflict of interest: The authors have declared that no conflict of interest exists.

Citation for this article: J. Clin. Invest. 118:2681-2684 (2008). doi:10.1172/JCI36515. mately 30-50 million people, rivaling the prevalence of cancer (4). However, the study of other, more rare hereditary macular diseases has also yielded fundamental knowledge that has greatly advanced our understanding of the molecular bases of vision. Historically, many of these major studies were published in 2 phases: the genetic association data was followed by insights into the functional implications of an identified polymorphism obtained via the use of transgenically engineered mice. In this issue of the JCI, Yang et al. give us the best of both worlds by presenting a combined functional genetics investigation of the critical nature of prominin 1 (PROM1; also known as $C D 133$ and $A C 133$ ) expression during photoreceptor disk morphogenesis that provides essential insight into the molecular programming of disk formation and the ever-expanding roles for PROM1 (5).

PROM1 is still best known for its original use as a human stem cell-specific marker (6), yet its known biological functions continue to reach far beyond this role. The protein is constructed of 5 transmembrane domains, 2 large extracellular loops containing $8 \mathrm{~N}$-linked glycosylation sites, and a cytoplasmic tail. Variable glycosylation

\section{Discovery of PROM1}

of these extracellular loops may account for the monoclonal antibody specificity for certain tissue types and circulating stem cells. Contemporaneous with the characterization of AC133 for hematopoietic cell lineage analysis, another group reported the discovery of a mouse protein, termed PROM1, found to be expressed on specific embryonic and adult epithelia and localized to plasma membrane protrusions (7). Although it was quickly realized in an exchange of public letters by the 2 laboratories that the human stem cell marker was the likely homolog of mouse PROM1, with more than $60 \%$ sequence overlap, an entire body of literature emerged in which the antigen was used to identify specific cell populations. In a recent JCI article, previously unchallenged claims that PROM1 was a marker of tumor-initiating metastatic colon cancer cells were rebutted in a study that demonstrated the initiation of colon cancer tumors in xenografts by PROM1-negative cells (8). Thus, it appears that PROM1 is not as lineage specific or functionally determined as it once was purported to be.

\section{PROM1 mutations are associated with hereditary macular degeneration}

There is mounting evidence that PROM1 is critical to the organization of photoreceptor disks. In 2000, a group that included members from the team that initially described mouse PROM1 found a genetic association between a human PROM1 frameshift mutation and a form of autosomal-recessive retinal degeneration in a small Indian pedigree (9). This polymorphism resulted in premature termination of the protein, which prohibited it from 


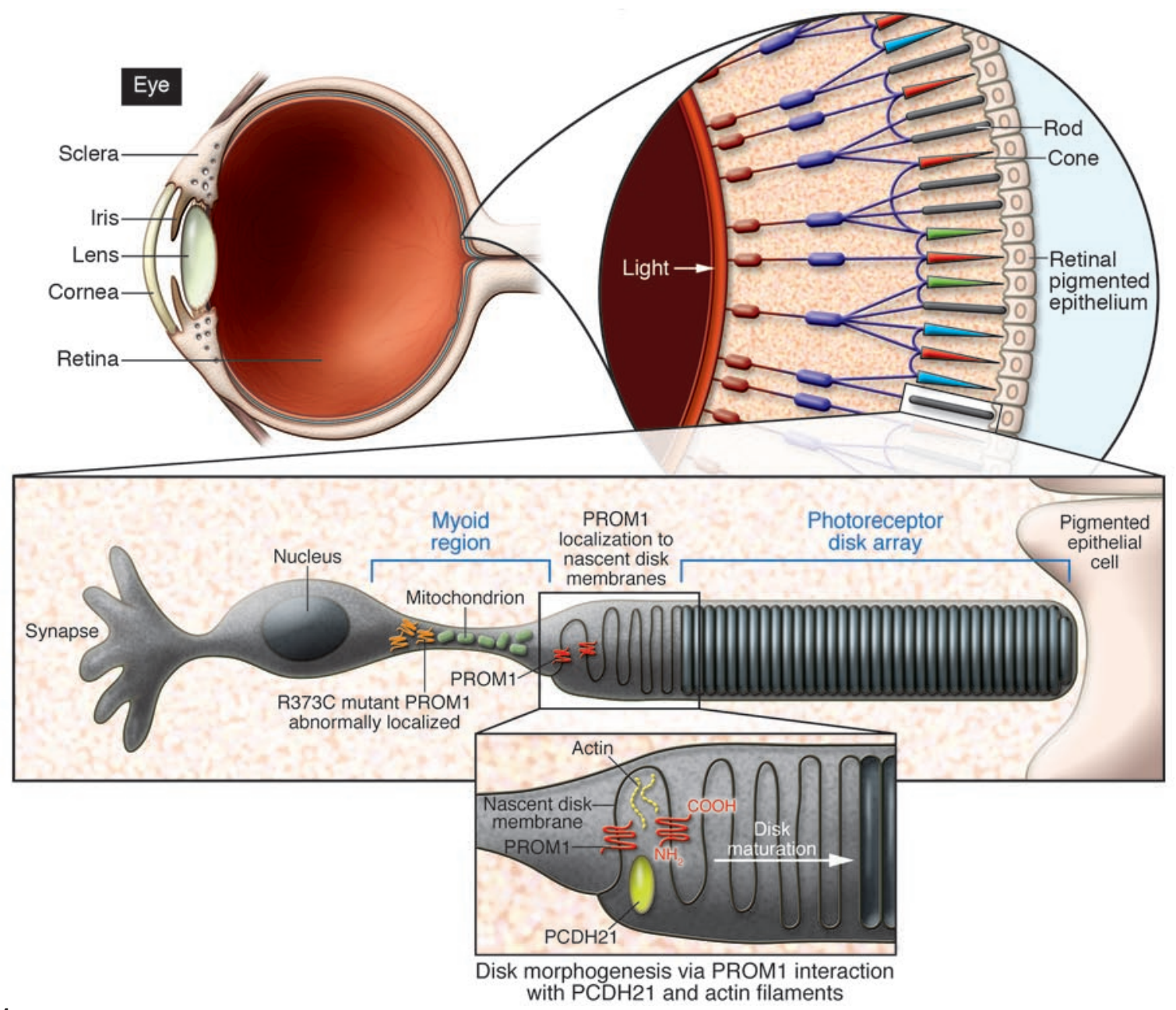

Figure 1

Retinal rod photoreceptor disk assembly requires PROM1. The rod photoreceptor cell consists of an outer segment containing an array of rhodopsin-loaded disks, a myoid region containing mitochondria, nucleus, and other organelles, and a synapse region that connects to the neural retinal network to transmit visual stimuli. PROM1 normally localizes to the nascent disk membranes, but in the case of the existence of an R373C mutation, the protein remains in the myoid region. In their study in this issue of the $\mathrm{JCl}$, Yang et al. show that PROM1 interacts with protocadherin 21 $(\mathrm{PCDH} 21)$ and actin filaments to regulate disk morphogenesis and subsequent maturation from evaginating nascent disk membranes (5).

reaching the cell surface. In these studies, PROM1 was found to localize to the base of the outer segment of murine rod photoreceptors, where disk biogenesis occurs. Yet the precise implications of this focused expression remained undefined. Yang et al. now provide us with critical information regarding the functionality of PROM1 in photoreceptor disk formation through an integrated approach that elegantly couples genetic association data with an in vivo animal study (5)

After identifying 2 pedigrees with different forms of inherited autosomal-dominant macular degeneration, the team mapped the 2 phenotypes to a region on chromosome $4 \mathrm{p}(5)$. In further genetic screening analyses of this region, a missense mutation was found in the coding sequence of the PROM1 gene that resulted in the replacement of arginine with cysteine at amino acid position 373 (R373C). Importantly, mutation analyses of a third pedigree with an autosomal-dominant cone-rod dystrophy also revealed the $\mathrm{R} 373 \mathrm{C}$ polymorphism, demonstrating that the PROM1 mutation is linked to 3 forms of dominant macular degeneration in humans.

To shed light on the biological effects of $\mathrm{R} 373 \mathrm{C}$ in vivo, transgenic mice were engineered containing either the wild-type or the R373C mutated human PROM1 gene under the control of the rhodopsin promoter, thereby localizing expression to the rod photoreceptors (5). In mice with the mutant PROM1, serial retinal imaging exhibited findings consistent with those found in humans. Progressive photore- ceptor degeneration was evident both in histological analyses and in analysis by electroretinography, a functional modality commonly used to quantify retinal response to light. The electron microscopy studies revealed a much more significant finding from this paper, that PROM1 appears to direct the organization of photoreceptor disks. Mice expressing the mutant PROM1 gene had malpositioned and overgrown disk membranes. As has been proposed elsewhere (10), PROM1 may be responsible for proper nascent disk alignment into bilayers (Figure 1). This mechanism is suggested by the presence of extracellular, leucine-like zipper motifs and the potential for PROM1 dimerization to link plasma membrane protrusions. These observations open a new avenue for 
investigation in the search for a molecular explanation of photoreceptor disk morphogenesis, a longstanding question in photoreceptor cell biology in particular and developmental biology in general.

\section{PROM1 is critical for photoreceptor disk biogenesis}

The study by Yang et al. (5) is a timely contribution to the field of retinal cell biology because it supports a hypothesis on photoreceptor disk formation that gained acceptance over the past 4 decades but has recently been challenged. Critical data presented here and elsewhere suggest that PROM1 is localized to the base of the outer segment and that without its functional presence, erroneous disk formation occurs. These findings align with a model of disk biogenesis wherein the outer segment base serves as the membrane source for disk renewal, a concept that has been supported by numerous other investigators. In 1964, a student from Sjöstrand's lab published an electron microscopy study of amphibian retina showing evaginations of the cell membrane of the photoreceptor outer segment (11). Several scientific giants went on to pioneer this field, including Richard Young, who rigorously studied the ultrastructure of photoreceptor elements in monkeys (12), and Roy Steinberg, who proposed an open model of disk biogenesis consisting of 2 membrane growth phases: evagination of the ciliary plasma membrane and formation of the disk rim (13). Further molecular work began to unravel the kinetics of disk formation and shedding $(14,15)$ as well as the protein interactions required for disk rim formation during photoreceptor membrane evagination (16). Now Yang et al. make another considerable stride by demonstrating that interactions among PROM1, protocadherin 21, and cytoskeletal actin regulate the outgrowth of evaginations of the plasma membrane of photoreceptor cilia, further supporting the open model of disk membrane formation (5).

In a significant departure from this hypothesis, a recent paper in Cell presented data suggesting that disks grow by fusion between opsin-containing vesicles and nascent disk membranes (17). Yang et al. argue strongly against this hypothesis with molecular and mutant phenotypic evidence that dually supports the earlier model (5). The fusion model has generated much skepticism because it is contradicted by an enormous cache of data showing nascent membrane formation at the base of the outer segment and the currently accepted open disk model of cone photoreceptor ultrastructure. The controversial data advancing the fusion model may be due to the choice of fixation agent, acrolein, which can significantly alter photoreceptor membranes (18) as well as 3 -dimensional skew as a result of section orientation. Regardless of the potential shortfalls, in this era of advancing molecular imaging, a conclusive resolution should be attainable. To date, freeze fracture studies of photoreceptor membranes have not provided a definitive ruling regarding the validity of the fusion model (19), but to our knowledge, a rigorous electron microscopic analysis of cryopreserved eyes has not been reported. Such a study visualizing the unperturbed membranes of the photoreceptor disk might unequivocally capture the functional morphology. Emerging 3-dimensional electron microscopy technologies could be leveraged to address this question. An alternative approach may be to use probes that can target the photoreceptor membrane and be secondarily labeled for imaging by electron microscopy or other new nanometer resolution systems. One such probe is a recently described actin-binding oligopeptide capable of nondestructive live visualization of cytoskeletal dynamics in vivo (20).

In conclusion, the discovery of PROM1associated macular degenerations simultaneously reveals an important molecular mechanism for photoreceptor disk formation and widens the biological ambit of PROM1 (5). In nature's mind, it would be woefully inefficient to create a unifunctional molecule. The growing recognition of PROM1's functional diversity attests to this notion and invites the continued investigation of its physiologic and clinical importance. It is also critical to take into account the gamut of biological roles for PROM1, especially given that targeted therapeutics are currently being developed for the treatment of some PROM1-expressing cancers (21).

\section{Acknowledgments}

The authors' research is generously supported by the Dr. E. Vernon Smith and Eloise C. Smith Macular Degeneration Research Endowed Chair, a Burroughs Wellcome Fund Clinical Scientist Award in Translational Research, Research to Prevent Blindness Lew R. Wassermann Merit and Physician Scientist Awards, the American Health Assistance Foundation, a
University of Kentucky University Research Professorship, and NIH grants EY015422, EY018350, and EY18836 (to J. Ambati) as well as by the International Retinal Research Foundation Charles D. Kelman Award (to M.E. Kleinman).

Address correspondence to: Jayakrishna Ambati, Department of Ophthalmology and Visual Sciences, E300 Kentucky Clinic, 740 S. Limestone Street, Lexington, Kentucky 40536-0284, USA. Phone: (859) 323-0686; Fax: (859) 323-1122; E-mail: jamba2@email.uky.edu.

1. Sjostrand, F.S. 1953. The ultrastructure of the outer segments of rods and cones of the eye as revealed by the electron microscope. J. Cell Physiol. 42:15-44.

2. Lamb, T.D., Collin, S.P., and Pugh, E.N., Jr. 2007. Evolution of the vertebrate eye: opsins, photoreceptors, retina and eye cup. Nat. Rev. Neurosci. 8:960-976.

3. Ambati, J., Ambati, B.K., Yoo, S.H., Ianchulev, S., and Adamis, A.P. 2003. Age-related macular degeneration: etiology, pathogenesis, and therapeutic strategies. Surv. Ophthalmol. 48:257-293.

4. Smith, W., et al. 2001. Risk factors for age-related macular degeneration: Pooled findings from three continents. Ophthalmology. 108:697-704.

5. Yang, Z., et al. 2008. Mutant prominin 1 found in patients with macular degeneration disrupts photoreceptor disk morphogenesis in mice. J. Clin. Invest. 118:2908-2916.

6. Yin, A.H., et al. 1997. AC133, a novel marker for human hematopoietic stem and progenitor cells. Blood. 90:5002-5012.

7. Weigmann, A., Corbeil, D., Hellwig, A., and Huttner, W.B. 1997. Prominin, a novel microvillispecific polytopic membrane protein of the apical surface of epithelial cells, is targeted to plasmalemmal protrusions of non-epithelial cells. Proc. Natl. Acad. Sci. U. S. A. 94:12425-12430.

8. Shmelkov, S.V., et al. 2008. CD133 expression is not restricted to stem cells, and both CD133 and CD133 metastatic colon cancer cells initiate tumors. J. Clin. Invest. 118:2111-2120.

9. Maw, M.A., et al. 2000. A frameshift mutation in prominin (mouse)-like 1 causes human retinal degeneration. Hum. Mol. Genet. 9:27-34.

10. Jászai, J., Fargeas, C.A., Florek, M., Huttner, W.B., and Corbeil, D. 2007. Focus on molecules: Prominin-1 (CD133). Exp. Eye Res. 85:585-586.

11. Nilsson, S.E. 1964. Receptor cell outer segment development and ultrastructure of the disk membranes in the retina of the tadpole (Rana pipiens). J. Ultrastruct. Res. 11:581-602.

12. Young, R.W. 1971. Shedding of discs from rod outer segments in the rhesus monkey. J. Ultrastruct. Res. 34:190-203.

13. Steinberg, R.H., Fisher, S.K., and Anderson, D.H. 1980. Disc morphogenesis in vertebrate photoreceptors. J. Comp. Neurol. 190:501-508.

14. Matsumoto, B., and Besharse, J.C. 1985. Light and temperature modulated staining of the rod outer segment distal tips with Lucifer yellow. Invest. Ophthalmol. Vis. Sci. 26:628-635.

15. Laties, A.M., Bok, D., and Liebman, P. 1976. Procion yellow: a marker dye for outer segment disc patency and for rod renewal. Exp. Eye Res. 23:139-148.

16. Arikawa, K., Molday, L.L., Molday, R.S., and Williams, D.S. 1992. Localization of peripherin/rds in the disk membranes of cone and rod photoreceptors: relationship to disk membrane morphogenesis and retinal degeneration. J. Cell Biol. 116:659-667. 
17. Chuang, J.Z., Zhao, Y., and Sung, C.H. 2007. SARAregulated vesicular targeting underlies formation of the light-sensing organelle in mammalian rods. Cell. 130:535-547.

18. Townes-Anderson, E. 1995. Intersegmental fusion in vertebrate rod photoreceptors. Rod cell structure revisited. Invest. Ophthalmol. Vis. Sci. 36:1918-1933.

19. Andrews, L.D., and Cohen, A.I. 1983. Freeze-fracture studies of photoreceptor membranes: new observations bearing upon the distribution of cholesterol. J. Cell Biol. 97:749-755.

20. Riedl, J., et al. 2008. Lifeact: a versatile marker to visualize F-actin. Nat. Methods. doi:10.1038/ nmeth.1220.

21. Smith, L.M., et al. 2008. CD133/prominin-1 is a potential therapeutic target for antibody-drug conjugates in hepatocellular and gastric cancers. Br. J. Cancer. doi:10.1038/sj.bjc.6604437.

\title{
Marking a path to transplant tolerance
}

\author{
Vicki Seyfert-Margolis ${ }^{1,2}$ and Laurence A. Turka ${ }^{2,3}$
}

\begin{abstract}
${ }^{1}$ Department of Medicine, University of California, San Francisco, San Francisco, California, USA. 2Immune Tolerance Network, Bethesda, Maryland, USA. ${ }^{3}$ Department of Medicine, University of Pennsylvania School of Medicine, Philadelphia, Pennsylvania, USA.
\end{abstract}

\begin{abstract}
Long-term allograft survival requires lifelong immunosuppression, which comes with serious side effects. Inducing immune tolerance to the transplant would enable immunosuppression withdrawal and revolutionize the quality of life of transplant recipients. In this issue of the JCI, MartinezLlordella et al. identify a profile of biomarkers that predict tolerance in liver transplant recipients (see the related article beginning on page 2845). These findings translate into a new means for prospectively selecting liver transplant patients who would benefit from immunosuppression withdrawal and ultimately may guide development of tolerogenic therapies that allow for allograft acceptance without the use of long-term immunosuppression.
\end{abstract}

The road to solid organ transplant tolerance may be somewhat shortened with the discovery of several new biomarkers for tolerance, as reported by Martínez-Llordella et al. in this issue of the JCI (1). These studies represent a significant advance in the ongoing effort to wean liver transplant recipients off immunosuppressive drugs. While newer immunosuppression protocols have vastly improved acute rejection rates in solid organ transplantation over the past 20 years, success is still not without its price - the consequences of longterm immunosuppression, often resulting in renal toxicity, opportunistic infections, and/or lymphoproliferative disease, remain significant clinical concerns. Clearly then, the idea that select liver transplant recipients might withdraw from all immunosuppression with little or no risk to their allograft deserves attention.

\section{Tolerance occurs in liver transplantation}

The liver has long been appreciated to be a relatively immunoprivileged organ. For example, in some rodent models of transplantation, liver grafts are often spontaneously accepted without a need for any

Conflict of interest: L.A. Turka has equity in GlaxoSmithKline.

Citation for this article: J. Clin. Invest. 118:2684-2686 (2008). doi:10.1172/JCI36552. immunosuppression, and it has been proposed that unique populations of antigenpresenting cells and ECs that reside in the liver are responsible for this phenomenon $(2,3)$. Studies of liver transplant recipients dating back to 1997 demonstrated that small numbers of patients could cease all immunosuppressive medications and still maintain a healthy graft (4). This observation triggered a series of studies in which the prospective withdrawal of immunosuppression was attempted in small cohorts of liver transplant recipients. The first such study was performed at the University of Pittsburgh, where 19\% of patients $(n=37)$ became drug free for at least 1 year (5). Subsequent studies in Japan and in the United Kingdom revealed that prospective weaning could be achieved in approximately $20 \%$ of enrolled patients. In these cases, weaning was successful in a total of 18 patients in the United Kingdom (6) and in a larger number in Japan $(7,8)$. More recently, the Immune Tolerance Network has established its support of a study of immunosuppression withdrawal in pediatric, parent-to-child living-donor liver transplant recipients. Overall, these studies and others have led to the generally accepted estimate that $20 \%$ of livertransplanted patients may be successfully withdrawn from immunosuppression.

That $20 \%$ of patients might be spared the risks of long-term immunosuppres- sion compels us to ask the question, can we identify these subjects a priori? One might similarly ask, can we do better than $20 \%$ ? The answer to both of these questions lies in defining biomarkers that indicate a propensity for successful immunosuppression withdrawal and that more clearly define the state of allograft tolerance.

\section{Can we predict tolerance?}

In their current study, Martínez-Llordella, et al. appear to have taken us a major step forward by providing a relatively small set of robust markers that can distinguish tolerant from nontolerant liver transplant recipients and from healthy individuals (1). Using a combination of quantitative real-time PCR and flow cytometry techniques, the authors point to increases in the numbers of peripheral T cells using the $\gamma \delta$ antigen receptor, in particular those expressing the $\delta 1$ form of the receptor, and to a difference in the activation state of circulating NK cells, as shown by a small set of differentially expressed genes. Critically, the predictive biomarkers were derived using a training set of samples and then validated in an independently gathered cohort of test-set patients. This work complements previously published reports of increased $\delta a-\gamma \delta \mathrm{TCR}^{+} \mathrm{T}$ cells in the blood of tolerant liver transplant patients $(7,9,10)$, thereby giving further credence to this measure as a valid biomarker of tolerance in this setting. Importantly, given the relative ease with which these assays can be performed and their targeted list of differentially expressed genes (26 in total), these assessments can be easily validated for use in the clinic (Figure 1).

\section{Do predictive markers tell us about mechanism?}

The proposed biomarkers (1) also raise important biological questions: What do the results from tolerant liver transplant 